\title{
Spontaneous hypothermia ameliorated inflammation and neurologic deficit in rat cardiac arrest models following resuscitation
}

\author{
MINGGEN ZHOU ${ }^{1,2 *}$, PENG WANG ${ }^{2,3 *}$, ZHENGFEI YANG ${ }^{2,3}$, HAIDONG WU $^{2,3}$ and ZITONG HUANG ${ }^{2,3}$ \\ ${ }^{1}$ Department of Intensive Care Unit; ${ }^{2}$ Institute of Cardiopulmonary Cerebral Resuscitation; ${ }^{3}$ Department of Emergency \\ Medicine, Sun Yat-sen Memorial Hospital, Sun Yat-sen University, Guangzhou, Guangdong 510120, P.R. China
}

Received July 15, 2016; Accepted June 14, 2017

DOI: $10.3892 / \mathrm{mmr} .2017 .8113$

\begin{abstract}
Cardiac arrest (CA) is a leading cause of mortality worldwide. The majority of the associated mortalities are caused by post-CA syndrome, which includes symptoms, such as neurologic damage, myocardial dysfunction and systemic inflammation. Following CA, return of spontaneous circulation (ROSC) leads to a brain reperfusion injury, which subsequently causes adverse neurologic outcomes or mortality. Therefore, investigating the underlying mechanisms of ROSC-induced neurologic deficits and establishing potential treatments is critical to prevent and treat post-CA syndrome. In the current study, CA rat models were established by asphyxia. Following ROSC, the temperature was controlled to achieve hypothermia. The general neurologic status was assessed using the neurologic deficit scale. Changes in the concentrations of interleukin (IL)-18 and IL-1 $\beta$ were measured with ELISA and the dynamic change in NACHT, LRR and PYD domains-containing protein 3 inflammasome components was determined by western blot analysis and immunohistochemistry. Neuronal death and apoptosis were measured via TUNEL assays. In the CA rat models, increasing the duration of CA before cardiopulmonary resuscitation was found to aggravate the neural deficit and increase the incidence of inflammation. Following ROSC, the expression level of the inflammasome components was observed to increase in CA rat models, which was accompanied by increased secretion of IL-18 and
\end{abstract}

Correspondence to: Dr Zhengfei Yang or Dr Zitong Huang, Department of Emergency Medicine, Sun Yat-sen Memorial Hospital, Sun Yat-sen University, 107 Yanjiang Xi Lu, Guangzhou, Guangdong 510120, P.R. China

E-mail: yangzhengfei@vip.163.com

E-mail: zithuang@163.com

*Contributed equally

Key words: cardiac arrest, return of spontaneous circulation, inflammasome, NACHT, LRR and PYD domains-containing protein 3 , spontaneous hypothermia, neurologic deficit
IL-1 $\beta$, indicating the promotion of inflammation. In addition, the study identified the beneficial role of spontaneous hypothermia in ameliorating the ROSC-induced inflammation and neurologic deficit in CA rat models, including the downregulation of inflammasome components and attenuating neuronal apoptosis. The present study contributes to the understanding of underlying mechanisms in CA-evoked inflammation and the subsequent neurologic damage following ROSC. A novel potential therapeutic strategy that may increase survival times and the quality of life for patients suffering from post-CA syndrome is proposed in the present study.

\section{Introduction}

Cardiac arrest (CA), also termed cardiopulmonary arrest, is a sudden stop in effective blood circulation, due to the failure of the heart to effectively contract or to contract at all. CA remains a major public health issue and is the most common direct cause of mortality in Western and developing countries $(1,2)$. Overall survival of cardiac arrest patients outside of a hospital setting is only $\sim 6.4 \%$ (3). This low rate is due to the initial difficulty in restoring hemodynamic stability (4), as well as to a high incidence of severe neurologic deficits caused by the CA (5). Therefore, $<30 \%$ of survivors are able to return to a normal functioning lifestyle (6).

The brain injury that results from the return of spontaneous circulation (ROSC) is a complicated process. The overall mechanisms remain elusive, but include altered $\mathrm{Ca}^{2+}$ homeostasis, free radical formation, mitochondrial dysfunction, protease activation, altered gene expression and inflammation $(7,8)$. These then lead to neuronal death in the central nervous system. Among all the potential mechanisms, inflammation stands out, as it is correlated with postischemic neuronal apoptosis (9). NACHT, LRR, and PYD domains-containing protein 3 (NLRP3), a component of the inflammasome, has been reported to function as a pathogen recognition receptor that recognizes pathogen-associated molecular patterns $(10,11)$. The inflammasome complex is a central component of the innate immune response via regulation of interleukin (IL)-1 $\beta$, IL-18 and pyroptosis (12). NLPR3 is significant in ischemia-reperfusion injuries in many tissue types, including renal (13), brain (14) and spinal cord (15). In the cerebral cortex, activation of the 
NLRP3-inflammasome induces the processing and release of IL-1 $\beta$ and IL-18 (16). Deletion of NLRP3 may ameliorate the neurovascular damage in ischemic stroke mice (17). Therefore, ischemic neural deficits caused by CA may also be mitigated via inhibition of NLRP3.

Hypothermia is defined as a body core temperature $<35.0^{\circ} \mathrm{C}$ and has been known to be a potent putative neuroprotectant (18). Mild hypothermia $\left(33^{\circ} \mathrm{C}\right)$ inhibits ischemia-induced promotion of mitochondrial membrane permeability, which may provide neuroprotection against cerebral injury following CA (19). Spontaneous hypothermia ( $\mathrm{SH})$ is clinically associated with the risk of mortality (20). Hickey et al (21) demonstrated that rats resuscitated from asphyxial CA developed neuroprotective SH (21). However, whether the neural deficit resulting from CA was correlated with activation of the inflammasome and was ameliorated by $\mathrm{SH}$ remains unclear. In the present study, the neural damage alterations and inflammasome component expression levels were elucidated following ROSC in established CA rat models. In addition, the role of $\mathrm{SH}$ in the inhibition of inflammasome component expression, and the amelioration of the neurologic deficit and neuronal death in the cerebral cortex were determined. The aim of the present study was to investigate the underlying mechanisms of ROSC-induced neurological deficits, and assess the potential strategies for the prevention and treatment of post-CA syndrome.

\section{Materials and methods}

Grouping. In total, 84 male specific pathogen-free grade Sprague Dawley rats (weight, 350-400 g; age, 8 weeks) were purchased from the Laboratory Animal Center of Guangzhou University of Chinese Medicine (Guangzhou, China). Firstly, 18 rats were randomly separated into three groups as follows: Group I, control group; Group II, the CA model group that received cardiopulmonary resuscitation (CPR) 4 min after CA; and Group III, the CA model group, which received CPR 6 min after CA. Secondly, 36 rats were then randomly separated into two groups, including 6 control rats and $30 \mathrm{CA}$ model rats. $\mathrm{CPR}$ was conducted $6 \mathrm{~min}$ after $\mathrm{CA}$. The CA rats were equally sub-divided into five groups, according to the different time points $(6,12,24,48$ and $72 \mathrm{~h})$ after ROSC. Thirdly, 30 rats were randomly divided into two groups, including six control rats and $24 \mathrm{CA}$ model rats. These $\mathrm{CA}$ rats were equally sub-divided into $\mathrm{SH}$ group and controlled normothermia $(\mathrm{CN})$ group. The $\mathrm{SH}$ and $\mathrm{CN}$ groups were again divided into two, determined by $24 \mathrm{~h}$ (SH Group I and CN Group I) or $48 \mathrm{~h}$ (SH Group II and CN Group II) after ROSC. The mortality rate of rats during the preparation of rat models was $6.7 \%$. The present study was approved by the ethics committee of Sun Yat-Sen University (Guangzhou, China).

CA rat model. Rat $\mathrm{CA}$ and resuscitation were performed, as previously described $(21,22)$. Anesthesia was achieved using $45 \mathrm{mg} / \mathrm{kg}$ pentobarbital sodium. Animals were ventilated with a Harvard Rodent Ventilator (Harvard Apparatus, Holliston, MA, USA), and the temperature was measured and maintained at $37 \pm 0.5^{\circ} \mathrm{C}$ throughout the preparation, insult, and first hour of recovery. Prior to asphyxia, the anesthetic gases were washed out with 3 min of ventilation at $100 \%$ oxygen, followed by 2 min of room air. Vecuronium was administered prior to asphyxia, in order to prevent reflex respiratory efforts during asphyxia. Subsequent to the washout period, animals were asphyxiated by disconnecting the respiratory tubing from the ventilator for $6 \mathrm{~min}$, resulting in $\sim 5 \mathrm{~min}$ of CA.

Resuscitation. Following exactly $5 \mathrm{~min}$, the ventilator was reconnected and ventilation was resumed with oxygen at a rate of 60 breaths $/ \mathrm{min}$. Intravenous epinephrine $(0.005 \mathrm{mg} / \mathrm{kg})$ and bicarbonate $(1.0 \mathrm{mEq} / \mathrm{kg})$ were administered, and external chest compressions were performed at a rate of 250-300 compressions/min. Rats generally experienced a ROSC within $2 \mathrm{~min}$. If not, an additional dose of epinephrine was administered. Following stabilization for at least $60 \mathrm{~min}$ and confirmation of adequate spontaneous respirations, rats were extubated and weaned from oxygen back to room air.

Temperature control. In the $\mathrm{CN}$ group, rats were maintained at $37 \pm 0.5^{\circ} \mathrm{C}$ during $\mathrm{CA}$ and for $4 \mathrm{~h}$ after resuscitation. In the $\mathrm{SH}$ group, rats were maintained at $37^{\circ} \mathrm{C}$ during CA, but following resuscitation were allowed to regulate their own temperature.

Neurologic deficit scale (NDS) score measurement. General neurologic status was assessed using the validated NDS at 24, 48, and $72 \mathrm{~h}$ after CA (23). The score includes an assessment of consciousness, respiration, cranial nerve activity, motor and sensory function, and coordination. Normal rats have an NDS of zero.

Enzyme-linked immunosorbent assay (ELISA). Arterial blood samples $(10 \mathrm{ml})$ were drawn at different time points after ROSC. Concentration changes in serum S100 calcium-binding protein B [S100B; cat. no. H6-KA0037, Multi Sciences (Lianke) Biotech Co., Ltd., Hangzhou, China], IL-1 $\beta$ [cat. no. 70-EK201B1/2, Multi Sciences (Lianke) Biotech Co., Ltd.] and IL-18 (cat.no. RK-KOA0362; Rockland Immunochemicals, Inc., Limerick, PA, USA) in the cerebral cortex were monitored by ELISA for the different groups. After coating with coating buffer $(50 \mathrm{mmol} / 1$ sodium carbonate buffer, $\mathrm{pH}=9.6)$, plates were sequentially washed with phosphate buffered saline with 0.05\% Tween-20 (PBST) buffer (cat. no. A100235-0001; Sangon Biotech Co., Ltd., Shanghai, China), blocked with $1 \%$ bovine serum albumin (BSA; cat. no. A602440-0050, Sangon Biotech Co., Ltd.), and incubated for $1 \mathrm{~h}$ at $37^{\circ} \mathrm{C}$. Anti-S100B (1:200), anti-IL-1 $\beta$ (1:200) or anti-IL-18 antibodies (1:100), and horseradish peroxidase-conjugated antibody $(1: 1,000$; all included in the corresponding ELISA kit) were sequentially added and incubated for $1 \mathrm{~h}$ at $37^{\circ} \mathrm{C}$. The chromogenic substrate 3,3',5,5'-Tetramethylbenzidine was added for detection. Absorbance was measured at a wavelength of $450 \mathrm{~nm}$ using an EnSpire multimode plate reader (Perkin Elmer, Waltham, Massachusetts).

Western blotting. Tissue samples were lysed in radioimmunoprecipitation buffer $(50 \mathrm{mM}$ Tris- $\mathrm{HCl}$ buffer $\mathrm{pH} \mathrm{7.4,}$ $150 \mathrm{mM} \mathrm{NaCl}, 5 \mathrm{mM}$ EDTA, $1 \% \mathrm{NP}-40$ and $0.25 \%$ sodium deoxycholate). Total protein was extracted from tissue lysate by centrifugation at $12,000 \mathrm{x} \mathrm{g}$ for $10 \mathrm{~min}$ at $4^{\circ} \mathrm{C}$ and protein concentrations were measured using a Bicinchoninic Acid assay (Sangon Biotech Co., Ltd.) according to the manufacturer's instructions. A total of $2 \mu \mathrm{g}$ protein was loaded onto each lane of 
Table I. Comparison of physiological parameters between Group I (Sham) and Groups II and III (the asphyxia groups).

\begin{tabular}{lcccr}
\hline Parameter & Group I $(\mathrm{n}=6)$ & Group II $(\mathrm{n}=6)$ & Group III $(\mathrm{n}=6)$ & P-value \\
\hline Body weight $(\mathrm{g})$ & $379.0 \pm 18.0$ & $376.0 \pm 16.0$ & $370.0 \pm 14.0$ & 0.7901 \\
Rectal temperature $\left({ }^{\circ} \mathrm{C}\right)$ & $36.9 \pm 0.3$ & $37.0 \pm 0.2$ & $37.0 \pm 0.2$ & 0.7008 \\
Mean arterial blood pressure (mmHg) & $110.0 \pm 7.0$ & $119.0 \pm 8.0$ & $109.0 \pm 9.0$ & 0.1033 \\
Heart rate (bpm) & $314.0 \pm 29.0$ & $310.0 \pm 29.0$ & $313.0 \pm 25.0$ & 0.9599 \\
Partial pressure of carbon dioxide & $36.3 \pm 2.8$ & $37.1 \pm 2.1$ & $37.9 \pm 2.4$ & 0.6253 \\
in end expiratory gas (mmHg) & & & &
\end{tabular}

Values are presented as mean \pm standard deviation.

$10 \%$ polyacrylamide gel (250V voltage for $2 \mathrm{~h}$ ) and blotted onto a polyvinylidene difluoride (PVDF) membrane. After blocking with PBST containing 5\% nonfat dry milk, the membrane was incubated with antibodies against NLRP3 (cat. no. 13158; 1:500), apoptosis-associated speck-like protein containing a CARD (ASC; cat. no. 67824; 1:500), caspase-1 (cat. no. 2225; 1:400), caspase-3 (cat. no. 9662; 1:500), and $\beta$-tubulin (cat. no. 2146; 1:500; all Cell Signaling Technology, Inc., Danvers, MA, USA). Peroxidase-linked anti rabbit IgG (Thermo Fisher Scientific, Inc., Waltham, MA, USA) served as a secondary antibody. These proteins were visualized using an ECL western blotting detection kit (GE Healthcare, Chicago, IL, USA).

Immunohistochemistry. The rats were anesthetized with an overdose of $150 \mathrm{mg} / \mathrm{kg}$ pentobarbital and then sacrificed. The whole brain was immediately removed and frozen on dry ice. Then, the cerebral cortex site was separated from the whole brain. The sections of the cerebral cortex $\left(\sim 1.5 \mathrm{~cm}^{3}\right)$ were sequentially washed in dimethylbenzene and ethanol, before being blocked in $3 \% \mathrm{H}_{2} \mathrm{O}_{2}$. All nonspecific binding sites were blocked for $30 \mathrm{~min}$ in PBS with 5\% BSA. The sections were then incubated at $4^{\circ} \mathrm{C}$ with anti-NLRP3, anti-ASC, anti-caspase-1, or anti-caspase-3 antibodies (Cell Signaling Technology, Inc.) overnight. Biotin-conjugated secondary antibody (cat. no. ab97044; Abcam, Cambridge, MA, USA) was applied to the slides and incubated for $1 \mathrm{~h}$ at room temperature. Finally, 3,3'-diaminobenzidine (DAB) $/ \mathrm{H}_{2} \mathrm{O}_{2}$ was added to the surface of the slide to develop the color at room temperature for $10 \mathrm{~min}$. The slides were visualized using a Nikon ECLIPSE 90i.

TUNEL assay. Sections were perfused with dimethylbenzene and sequentially washed with different concentrations of ethanol and blocked in $3 \% \mathrm{H}_{2} \mathrm{O}_{2}$. These sections were incubated in 5\% BSA for $30 \mathrm{~min}$ and the fragmented DNA was labeled with the TUNEL reaction solution at $37^{\circ} \mathrm{C}$ for $1 \mathrm{~h}$. Converter-peroxidase was added to the sections at $37^{\circ} \mathrm{C}$ for $30 \mathrm{~min}$ before the TUNEL-positive nuclei were visualized by adding the DAB staining solution. All images were captured using a Nikon ECLIPSE 90i.

Statistical analysis. Data are presented as means \pm standard error of the mean and analysis was performed with GraphPad Prism 6 software (GraphPad Software, Inc., La Jolla, CA, USA). The normalized intensity for western blotting bands was measured with Image J software version 1.45 (National Institutes of Health, Bethesda, MD, USA). Unpaired Student's t-tests and one-way ANOVA with a Tukey post hoc test were used to determine significant differences. $\mathrm{P}<0.05$ was considered to indicate a statistically significant difference.

\section{Results}

Increasing duration of CA aggravated neural defects. In order to elucidate the role of CA in neural defects, physiological parameters were compared between the control group and the two asphyxia groups, determining that there was no significant difference in physiological parameters among the three groups (Table I). Similarly, no apparent alteration in the majority of physiological parameters was observed between the two asphyxia groups following resuscitation. However, the duration of ROSC and the duration without blood flow in the asphyxia group that received CPR at 6 min after CA (Group III) was significantly longer than in the asphyxia group that received CPR at 4 min after CA (Group II; Table II). In addition, it was found that anal temperature was lower in the two asphyxia groups when compared with the control group. In addition, the rectal temperature in Group III was lower than that in Group II at five consecutive time points (from 10-120 min), but not at 180 min after ROSC (Fig. 1A).

Subsequently, it was determined whether the various neural defects were present in the asphyxia models. As shown in Fig. 1B, the NDS score was significantly lower in Group III than in Group II at 24, 48 and $72 \mathrm{~h}$ after CPR. Additionally, the S100B serum concentration of Group II and III was significantly greater than that in Group I at $72 \mathrm{~h}$ after CPR (Fig. 1C). The immunohistochemistry results indicated that cell morphology in the cerebral cortex was normal in the control (Group I; Fig. 1D). By contrast, morphological damage was observed in the cerebral cortex of Group II, including a vacuole and swelling in the cytoplasm (Fig. 1E). Further severe damage was observed in Group III, where necrosis was observed in the cells of cerebral cortex (Fig. 1F). These data demonstrated that increasing duration of CA increased the severity of neural damage in the cerebral cortex and enhanced the incidence of inflammation.

Dynamic expression patterns of NLRP3, ASC and caspase-1 following ROSC. As the observed neural defects were associated with inflammation, whether the expression levels of 
Table II. Comparison of physiological parameters between the two asphyxia groups prior to CPR.

\begin{tabular}{|c|c|c|c|}
\hline Groups & Group II (n=6) & Group III (n=6) & P-value \\
\hline Duration of asphyxia before CA (sec) & $190 \pm 14$ & $199 \pm 30$ & 0.5334 \\
\hline CPR time to ROSC (sec) & $94 \pm 27$ & $163 \pm 10^{\mathrm{a}}$ & $<0.001$ \\
\hline No flow time (sec) & $214 \pm 27$ & $540 \pm 15^{\mathrm{a}}$ & $<0.001$ \\
\hline \multicolumn{4}{|l|}{ Mean arterial blood pressure $(\mathrm{mmHg})$} \\
\hline 10 min post ROSC & $118 \pm 12$ & $109 \pm 18$ & 0.3947 \\
\hline 30 min post ROSC & $102 \pm 8$ & $117 \pm 16$ & 0.0561 \\
\hline 60 min post ROSC & $114 \pm 18$ & $104 \pm 16$ & 0.3558 \\
\hline 90 min post ROSC & $100 \pm 16$ & $113 \pm 10$ & 0.0555 \\
\hline 120 min post ROSC & $104 \pm 13$ & $105 \pm 19$ & 0.8802 \\
\hline \multicolumn{4}{|l|}{ Heart rate $(\mathrm{bpm})$} \\
\hline 10 min post ROSC & $311 \pm 41$ & $306 \pm 32$ & 0.7353 \\
\hline 30 min post ROSC & $295 \pm 18$ & $308 \pm 39$ & 0.4737 \\
\hline 60 min post ROSC & $313 \pm 28$ & $298 \pm 30$ & 0.3682 \\
\hline 90 min post ROSC & $288 \pm 22$ & $300 \pm 31$ & 0.4599 \\
\hline 120 min post ROSC & $306 \pm 27$ & $316 \pm 43$ & 0.625 \\
\hline \multicolumn{4}{|l|}{$\begin{array}{l}\text { Partial pressure of carbon dioxide in } \\
\text { end expiratory gas }(\mathrm{mmHg})\end{array}$} \\
\hline 10 min post ROSC & $44.5 \pm 27$ & $49 \pm 4.6$ & 0.0731 \\
\hline $30 \mathrm{~min}$ post ROSC & $65.1 \pm 5.7$ & $71.8 \pm 4.5^{\mathrm{b}}$ & 0.0454 \\
\hline 60 min post ROSC & $58.0 \pm 4.1$ & $57.3 \pm 4.7$ & 0.7632 \\
\hline 90 min post ROSC & $43.7 \pm 3.3$ & $45.6 \pm 3.2$ & 0.3342 \\
\hline 120 min post ROSC & $40.4 \pm 3.4$ & $40.3 \pm 2.4$ & 0.9616 \\
\hline
\end{tabular}

Group II, CPR was conducted 4 min after CA; Group III, CPR was conducted 6 min after CA. ${ }^{\mathrm{a} P}<0.01$, ${ }^{\mathrm{b}} \mathrm{P}<0.05$ vs. Group II. CPR, cardiopulmonary resuscitation; $\mathrm{CA}$, cardiac arrest; ROSC, return of spontaneous circulation.

certain key inflammatory factors were altered following resuscitation in the $\mathrm{CA}$ models were investigated. The potential changes in physiological parameters between the control and five asphyxia groups that were resuscitated at different time points after ROSC were detected. No apparent changes in physiological parameters were observed among the six groups, as presented in Table III. Furthermore, no significant difference in duration of asphyxia and resuscitation among the six groups was observed (Table IV). Whether the expression levels of key components of the inflammasome, including NLRP3, ASC and caspase-1 were altered following ROSC were determined. The expression level of NLRP3 was constantly demonstrated to increase at different time points, except for $48 \mathrm{~h}$ after ROSC (Fig. 2A and B). In addition, it was found that the expression level of ASC significantly increased at $6 \mathrm{~h}$ after ROSC, but did not exhibit any subsequent further increase (Fig. 2A and C). The expression level of caspase- 1 increased at 6 and $48 \mathrm{~h}$ after ROSC, but showed no further increase at 12, 24 or $72 \mathrm{~h}$ after ROSC compared with the previous time points (Fig. 2A and D). These findings were confirmed by immunohistochemical analysis. The number of ASC, NLRP3 and caspase-1 positive cells significantly increased at different time points after ROSC, compared with what was observed in the control (Fig. 3A). Collectively, these results indicate that the expression levels of NLRP3, ASC and caspase-1 increase with increasing time following ROSC, further enhancing inflammation.
Concentrations of IL-1 $\beta$ and IL-18 increased in the cerebral cortex after ROSC. ELISA assays were performed to determine whether the concentrations of IL-1 $\beta$ and IL-18 in the cerebral cortex increased after ROSC. Compared with the control group, the concentration of IL-1 $\beta$ was significantly higher at $6 \mathrm{~h}$ and reached the maximal level at $12 \mathrm{~h}$ after ROSC (Fig. 3B). The concentration of IL-1 $\beta$ concentration gradually decreased at 24 , 48 and $72 \mathrm{~h}$ after ROSC, but continued to be higher than that of the control group (Fig. 3B). Similarly, the IL-18 concentration was significantly higher after $6 \mathrm{~h}$, and consistently increased with increasing time after ROSC (Fig. 3C). These data demonstrated that IL-1 $\beta$ and IL-18 concentrations were elevated in CA rat models after ROSC, indicating the aggravation of inflammation in the cerebral cortex.

SH alleviated neurological deficiency, apoptosis and inflammation in the CA rat model. Subsequently, the effects of SH on the neurological deficiency and inflammation observed in CA models were elucidated. No significant differences in physiological parameters were observed between the control, SH and CN groups (Table V). In the CA models, no apparent alteration in asphyxia time and CPR to ROSC time was identified, nor was any change observed in the tested physiological parameters among all four asphyxia groups following ROSC, as presented in Table VI. By contrast, the rectal temperature was significantly lower in the $\mathrm{SH}$ group than in the $\mathrm{CN}$ group 
Table III. Comparison of physiological parameters among the sham group and five asphyxia groups at different time points after ROSC.

\begin{tabular}{|c|c|c|c|c|c|}
\hline Group (n) & Body weight (g) & $\begin{array}{c}\text { Rectal } \\
\text { temperature }\left({ }^{\circ} \mathrm{C}\right)\end{array}$ & $\begin{array}{c}\text { Mean arterial blood } \\
\text { pressure }(\mathrm{mmHg})\end{array}$ & $\begin{array}{l}\text { Heart rate } \\
\quad(\mathrm{bpm})\end{array}$ & $\mathrm{P}_{\mathrm{ET}} \mathrm{CO}_{2}(\mathrm{mmHg})$ \\
\hline Sham Group (6) & $375 \pm 18$ & $36.9 \pm 0.4$ & $115 \pm 12$ & $320 \pm 25$ & $35.73 \pm 2.0$ \\
\hline \multicolumn{6}{|l|}{ Time after ROSC } \\
\hline $6 \mathrm{~h}(6)$ & $376 \pm 16$ & $36.9 \pm 0.2$ & $109 \pm 16$ & $317 \pm 25$ & $37.0 \pm 2.1$ \\
\hline $12 \mathrm{~h}(6)$ & $374 \pm 16$ & $37.0 \pm 0.2$ & $110 \pm 10$ & $314 \pm 36$ & $37.7 \pm 2.5$ \\
\hline $24 \mathrm{~h}(5)$ & $368 \pm 19$ & $36.8 \pm 0.2$ & $106 \pm 14$ & $312 \pm 26$ & $38.0 \pm 2.7$ \\
\hline $48 \mathrm{~h}(6)$ & $368 \pm 15$ & $36.9 \pm 0.3$ & $113 \pm 10$ & $305 \pm 30$ & $38.3 \pm 2.1$ \\
\hline $72 \mathrm{~h}(6)$ & $383 \pm 15$ & $37.1 \pm 0.2$ & $109 \pm 13$ & $312 \pm 34$ & $38.2 \pm 3.2$ \\
\hline P-value & 0.5976 & 0.717 & 0.9287 & 0.9670 & 0.4426 \\
\hline
\end{tabular}

ROSC, return of spontaneous circulation; $\mathrm{P}_{\mathrm{ET}} \mathrm{CO}_{2}$, partial pressure of carbon dioxide in end expiratory gas.

Table IV. Comparison of asphyxia and CPR duration among the five asphyxia groups with different time points after ROSC ( $\mathrm{n}=6$ per group).

\begin{tabular}{lcc}
\hline Time after ROSC (h) & Asphyxia (sec) & CPR (sec) \\
\hline 6 & $189 \pm 32$ & $166 \pm 26$ \\
12 & $204 \pm 30$ & $175 \pm 33$ \\
24 & $215 \pm 27$ & $179 \pm 33$ \\
48 & $194 \pm 24$ & $168 \pm 27$ \\
72 & $182 \pm 26$ & $164 \pm 17$ \\
P-value & 0.4245 & 0.8821 \\
\hline
\end{tabular}

CPR, cardiopulmonary resuscitation; CA, cardiac arrest; ROSC, return of spontaneous circulation.

(Fig. 4A). The NDS score was significantly higher in the SH group compared with the $\mathrm{CN}$ group at 24 and $48 \mathrm{~h}$ after ROSC (Fig. 4B). Additionally, the S100B concentration was shown to be reduced in the $\mathrm{SH}$ group compared with the $\mathrm{CN}$ group at 24 and $48 \mathrm{~h}$ after ROSC (Fig. 4C). Western blot analysis demonstrated that the expression levels of NLRP3, ASC, caspase-1 and caspase- 3 markedly decreased in the $\mathrm{SH}$ groups compared with the CN group (Fig. 4D-H). Similar results were obtained via immunohistochemical analysis. The number of NLRP3, ASC, caspase-1 and caspase-3 positive cells in the SH group significantly decreased compared with the $\mathrm{CN}$ group (Fig. 5A). In addition, apoptosis was partially inhibited in the $\mathrm{SH}$ group, as the ratio of TUNEL-positive cells in this group was markedly reduced when compared with the $\mathrm{CN}$ group, while there was a limited number of apoptotic cells in the control group (Fig. 5B). Furthermore, SH after ROSC markedly decreased the concentrations of IL- $1 \beta$ and IL-18 (Fig. 5C). Finally, the cells in the cerebral cortex exhibited more severe damage in the CN group than did the cells in the $\mathrm{SH}$ group, while the cell morphology in the control group was normal (Fig. 5D). Thus, these data demonstrate that SH alleviated neural defects, apoptosis, and inflammation in the cerebral cortex, when compared with the $\mathrm{CN}$ group.

\section{Discussion}

The present study demonstrates that the expression levels of inflammasome components changed in CA rat models following ROSC, potentially indicating the participation of the inflammasome in post-CA syndrome. In addition, it was found that spontaneous hypothermia mitigated the neural defects and inflammation induced by CPR and ROSC in CA models compared with the $\mathrm{CN}$ model, providing mechanistic exploration of the effect of SH on post-CA syndrome and its potential correlation with the inflammasome.

Hypothermia is common in patients with neurologic disorders and those in a critical condition. In the present study, hypothermia was demonstrated in the established CA rat models. This is consistent with previous findings showing that rats resuscitated from asphyxial CA developed mild to moderate hypothermia (21). An important factor leading to hypothermia is the duration of the perfusion without blood flow. It was identified that a longer duration of asphyxia prior to resuscitation caused a lower body temperature and thus a more serious neural deficit. Hypothermia is a double-edged sword, however, as SH exerted a protective effect against neurologic damage and inflammation in the CA models. Until now, hypothermia has only been a validated effective treatment for brain resuscitation following CA. There is no consensus on the specific duration of hypothermia in the treatment, but the generally accepted time is $12-24 \mathrm{~h}(24,25)$. Theoretically, longer durations of hypothermia are more beneficial to the neural tissues. However, there are side effects during the treatment, such as prolonged clotting time and pulmonary infection. Consistent with the current finding in rat models, Vijlbrief et al (26) identified that hypothermia following perinatal asphyxia exerted a beneficial effect on cardiac function in infants, indicating its potential clinical application.

CA induced increased expression levels of pro-inflammatory cytokines, therefore, aggravating the inflammatory reaction. In the hypothermia treatment, however, expression levels of inflammation-associated components are altered. Callaway et al (27) identified that SH alleviated the NDS in CA models, but concluded that altering the inflammatory response subsequent to $\mathrm{CA}$ is not necessary to achieve the beneficial 
A

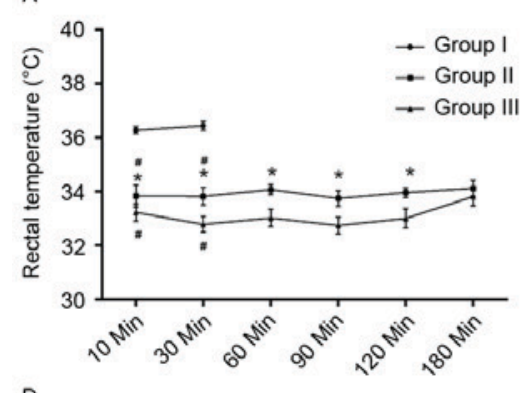

D

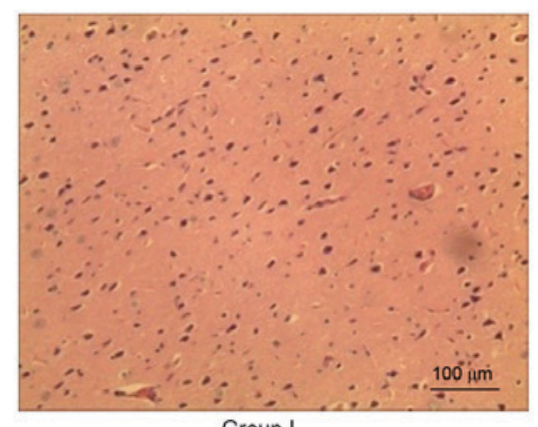

B

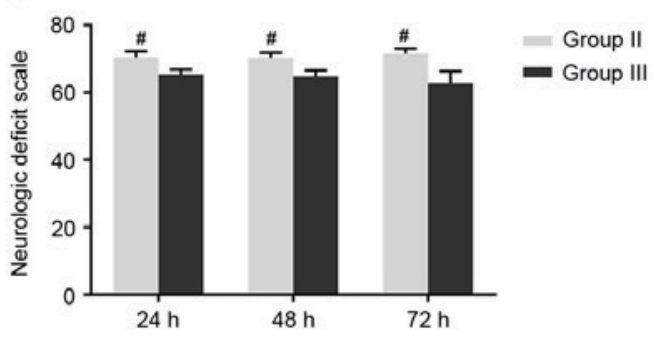

c

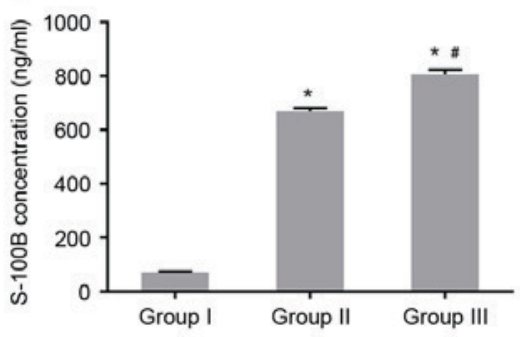

Figure 1. Increasing duration of asphyxia exacerbated neural damage in CA models. (A) The rectal temperature was lower in the asphyxia groups than in the control group, while Group II exhibited higher rectal temperatures than Group III. " $\mathrm{P}<0.05$ vs. Group III at the same time point; " $\mathrm{P}<0.05$ vs. Group I at the same time point (one-way ANOVA). (B) The neurologic deficit scale score in Group II was higher than that of Group III. "P<0.05 vs. Group III at the same time point (unpaired Student's t-test). (C) Among the three groups, the S100B concentration was highest in Group III and lowest in Group II. "P<0.01 vs. Group I; ${ }^{\text {\#}} \mathrm{P}<0.05$ vs. Group II (one-way ANOVA). (D) Immunohistochemistry demonstrated light damage in the control group, severe damage in Group II, and further severe damage in Group III. The statistical difference was determined by unpaired Student's t-test. CA, cardiac arrest; S100B, S100 calcium-binding protein B.

A

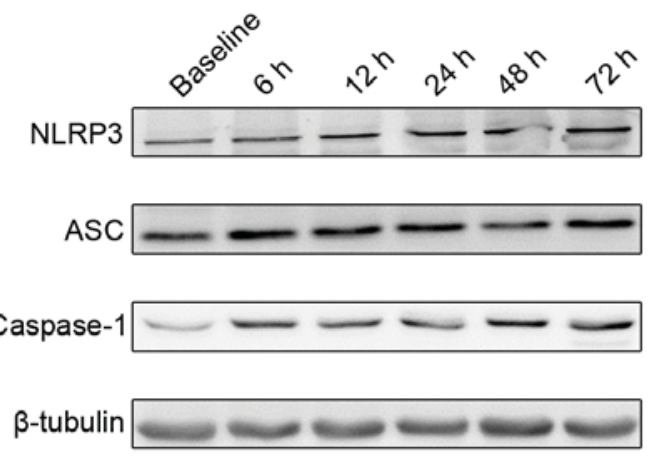

C

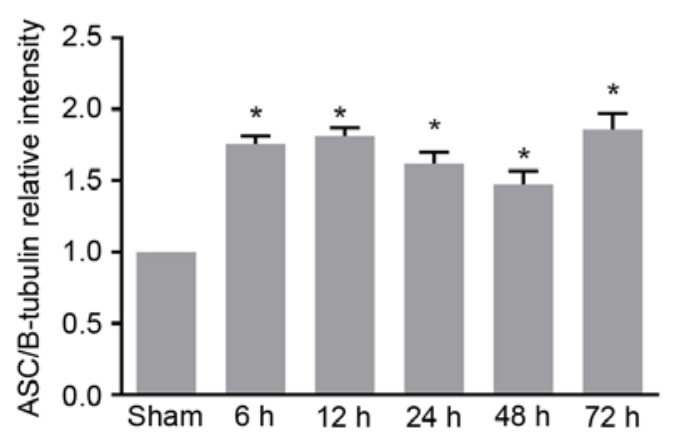

B

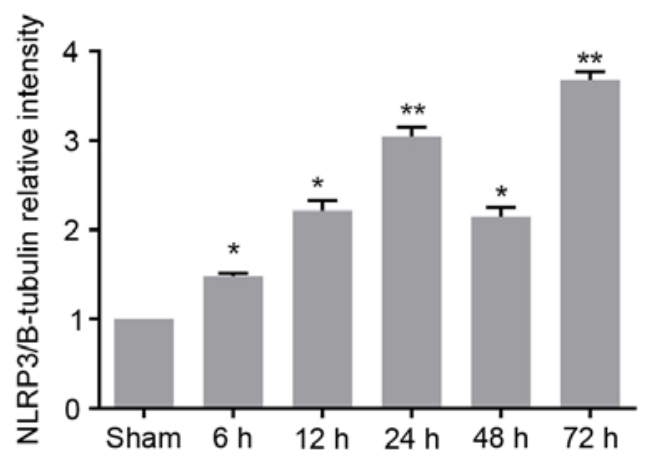

D

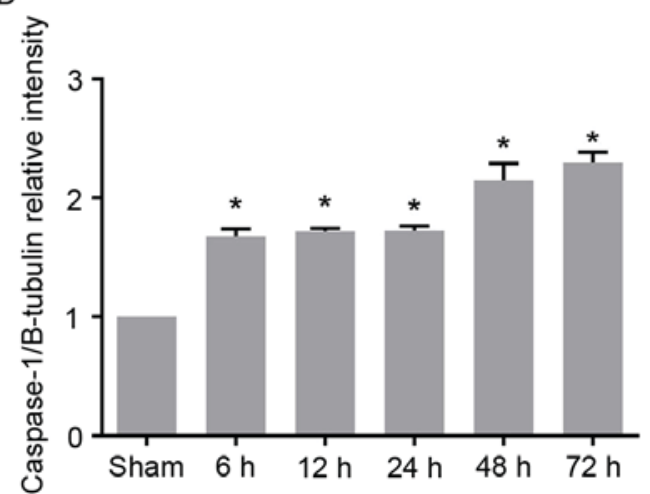

Figure 2. Change in the expression levels of inflammasome components following ROSC in the CA model. (A) The expression levels of NLRP3, ASC and caspase-1 increased at different time points following ROSC. Unpaired Student's t-test analysis of the relative intensity of (B) NLRP3, (C) ASC and (D) caspase-1 expression subsequent to ROSC in the CA model. " $\mathrm{P}<0.05$ and ${ }^{* *} \mathrm{P}<0.01$ vs. the control (Sham) group. ROSC, return of spontaneous circulation; CA, cardiac arrest; NLRP3, NACHT, LRR, and PYD domains-containing protein 3; ASC, apoptosis-associated speck-like protein containing a CARD. 
Table V. Comparison of physiological parameters among the sham group and asphyxia groups with SH and CN.

\begin{tabular}{|c|c|c|c|c|c|}
\hline Group & $\begin{array}{l}\text { Body weight } \\
\text { (g) }\end{array}$ & $\begin{array}{c}\text { Rectal } \\
\text { temperature }\left({ }^{\circ} \mathrm{C}\right)\end{array}$ & $\begin{array}{c}\text { Mean arterial blood } \\
\text { pressure }(\mathrm{mmHg})\end{array}$ & $\begin{array}{l}\text { Heart rate } \\
\quad(\mathrm{bpm})\end{array}$ & $\mathrm{P}_{\mathrm{ET}} \mathrm{CO}_{2}(\mathrm{mmHg})$ \\
\hline Sham (6) & $378 \pm 19$ & $37.0 \pm 0.3$ & $105 \pm 13$ & $320 \pm 22$ & $37.1 \pm 3.3$ \\
\hline SH Group I (6) & $385 \pm 10$ & $37.0 \pm 0.3$ & $113 \pm 13$ & $313 \pm 28$ & $36.7 \pm 1.9$ \\
\hline CN Group I (6) & $376 \pm 16$ & $36.9 \pm 0.3$ & $111 \pm 12$ & $312 \pm 25$ & $37.1 \pm 2.5$ \\
\hline SH Group II (6) & $376 \pm 21$ & $37.0 \pm 0.4$ & $107 \pm 17$ & $304 \pm 28$ & $36.4 \pm 1.9$ \\
\hline CN Group II (6) & $377 \pm 18$ & $37.0 \pm 0.3$ & $109 \pm 12$ & $310 \pm 32$ & $37.6 \pm 2.6$ \\
\hline P-value & 0.8626 & 0.8268 & 0.9287 & 0.8774 & 0.7148 \\
\hline
\end{tabular}

$\mathrm{P}_{\mathrm{ET}} \mathrm{CO}_{2}$, partial pressure of carbon dioxide in end expiratory gas; $\mathrm{SH}$, spontaneous hypothermia; $\mathrm{CN}$, controlled normothermia.

Table VI. Comparison of asphyxia and resuscitation duration, as well as physiological parameters among the four asphyxia groups $(n=6)$.

\begin{tabular}{|c|c|c|c|c|c|}
\hline \multirow[b]{2}{*}{ Group } & \multicolumn{2}{|c|}{ Group I } & \multicolumn{2}{|c|}{ Group II } & \multirow[b]{2}{*}{ P-value } \\
\hline & $\mathrm{SH}$ & $\mathrm{CN}$ & $\mathrm{SH}$ & $\mathrm{CN}$ & \\
\hline Asphyxia time (sec) & $190 \pm 30$ & $198 \pm 33$ & $206 \pm 38$ & $196 \pm 40$ & 0.8816 \\
\hline Cardiopulmonary resuscitation to ROSC time (sec) & $164 \pm 18$ & $180 \pm 35$ & $176 \pm 27$ & $175 \pm 34$ & 0.7839 \\
\hline \multicolumn{6}{|l|}{ Mean arterial blood pressure $(\mathrm{mmHg})$} \\
\hline 30 min post ROSC & $110 \pm 10$ & $125 \pm 10$ & $117 \pm 11$ & $116 \pm 15$ & 0.2102 \\
\hline 60 min post ROSC & $108 \pm 11$ & $115 \pm 12$ & $109 \pm 12$ & $119 \pm 14$ & 0.3488 \\
\hline 90 min post ROSC & $106 \pm 10$ & $105 \pm 13$ & $102 \pm 13$ & $110 \pm 16$ & 0.7597 \\
\hline 120 min post ROSC & $107 \pm 12$ & $108 \pm 11$ & $114 \pm 13$ & $117 \pm 18$ & 0.5464 \\
\hline \multicolumn{6}{|l|}{ Heart rate (bpm) } \\
\hline 30 min post ROSC & $310 \pm 34$ & $317 \pm 29$ & $316 \pm 32$ & $308 \pm 33$ & 0.9530 \\
\hline 60 min post ROSC & $310 \pm 30$ & $316 \pm 29$ & $307 \pm 25$ & $322 \pm 27$ & 0.7792 \\
\hline 90 min post ROSC & $309 \pm 32$ & $318 \pm 33$ & $320 \pm 29$ & $312 \pm 23$ & 0.9069 \\
\hline 120 min post ROSC & $317 \pm 40$ & $327 \pm 34$ & $306 \pm 27$ & $313 \pm 28$ & 0.7242 \\
\hline \multicolumn{6}{|l|}{$\mathrm{P}_{\mathrm{ET}} \mathrm{CO}_{2}(\mathrm{mmHg})$} \\
\hline 30 min post ROSC & $62.9 \pm 6.2$ & $68.2 \pm 4.3$ & $66.03 \pm 4.6$ & $70.00 \pm 4.2$ & 0.1060 \\
\hline 60 min post ROSC & $48.7 \pm 5.7$ & $46.1 \pm 6.8$ & $45.9 \pm 5.5$ & $47.9 \pm 6.7$ & 0.8226 \\
\hline 90 min post ROSC & $37.9 \pm 2.5$ & $36.9 \pm 2.8$ & $36.8 \pm 2.3$ & $39.6 \pm 3.6$ & 0.2945 \\
\hline 120 min post ROSC & $36.6 \pm 2.9$ & $38.60 \pm 3.2$ & $34.5 \pm 5.7$ & $38.1 \pm 3.0$ & 0.2784 \\
\hline
\end{tabular}

ROSC, return of spontaneous circulation; $\mathrm{P}_{\mathrm{ET}} \mathrm{CO}_{2}$, partial pressure of carbon dioxide in end expiratory gas; $\mathrm{SH}$, spontaneous hypothermia; $\mathrm{CN}$, controlled normothermia.

effects of hypothermia (27). By contrast, NLRP3 expression was demonstrated to be lower in the SH group compared with the $\mathrm{CN}$ group, and the secretion of IL-18 and IL-1 $\beta$ was decreased in the SH group. This discrepancy is likely due to the different techniques used to initiate the asphyxia and trigger the activation of the NLRP3 inflammasome. Activation of the NLRP3 inflammasome depends on an increase in ATP provision and calcium load, while hypothermia may inhibit the calcium influx and upload (28). Therefore, $\mathrm{SH}$ following resuscitation may work via the above-mentioned mechanisms to inhibit the NLRP3 inflammasome, and potentially downstream IL-18 and IL-1 $\beta$ expression, to exert a neuroprotective effect. However, the specific mechanisms involved require further investigation.
Cell death or apoptosis occurs following ischemia or reperfusion. Previous reports have indicated that hypothermia inhibits caspase-3 mRNA expression (29). In the caspase family, caspase-1 participates in the inflammatory reaction and is responsible for the activation of pro-inflammatory cytokines, while caspase-3 mediates apoptosis (30). In the present study, CA caused upregulation of the expression level of caspase-1 and caspase-3; further demonstrating that inflammation and apoptosis were promoted by CA. The initiation of apoptosis occurs subsequent to inflammation. There is, therefore, enough time to intervene before CA induces apoptosis. Various types of treatments such as hypothermia may inhibit apoptosis via caspase-3 inhibition. The current study identified 
A
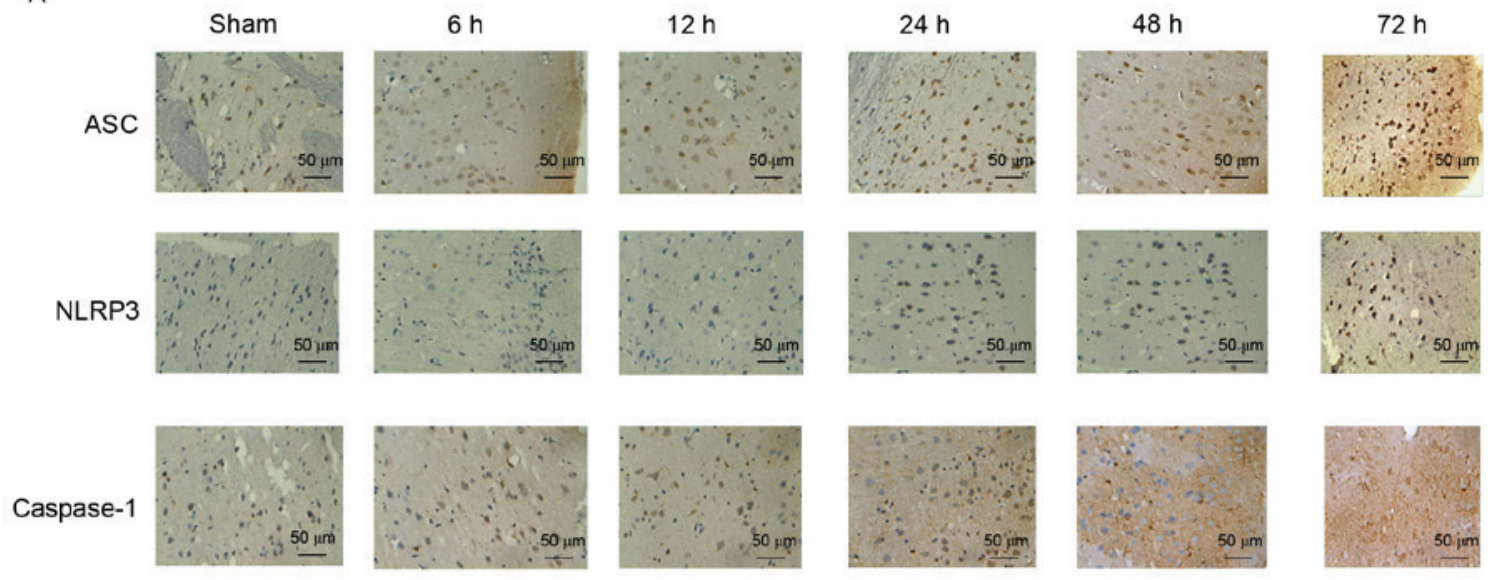

B

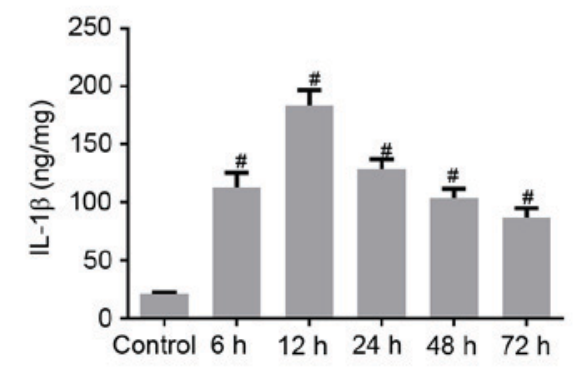

C

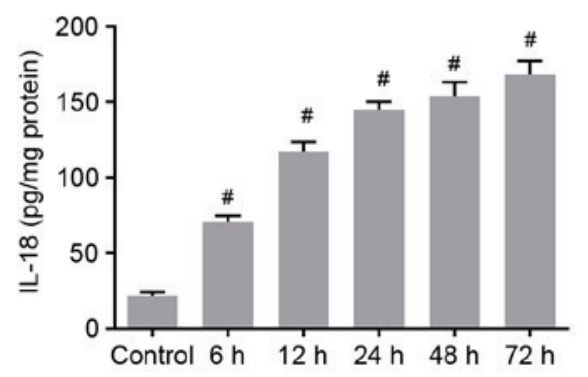

Figure 3. (A) Immunostaining analysis of ASC, NLRP3 and caspase-1 in the cerebral cortex of control rats and CA models at 6, 12, 24, 48, and 72 h after ROSC. Scale bar, $50 \mu \mathrm{m}$. The concentrations of (B) IL-1 13 and (C) IL-18 increased in the cerebral cortex of CA models following ROSC. "P<0.01 vs. the control group (unpaired Student's t-test). ASC, apoptosis-associated speck-like protein containing a CARD; NLRP3, NACHT, LRR, and PYD domains-containing protein 3; CA, cardiac arrest; ROSC, return of spontaneous circulation; IL, interleukin.
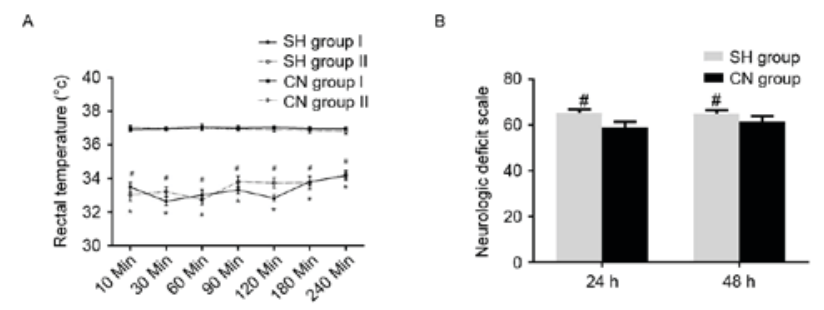

c

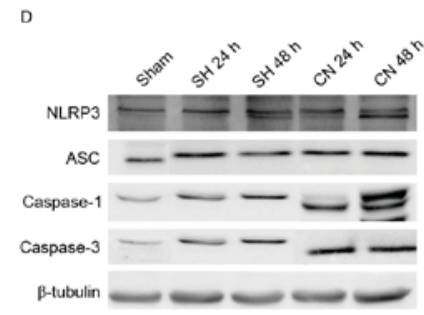

$\mathrm{E}$
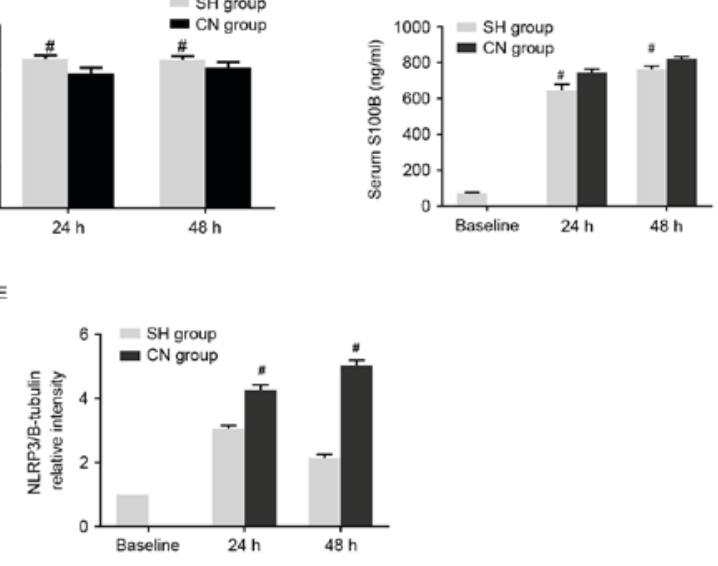

$\mathbf{F}$

G
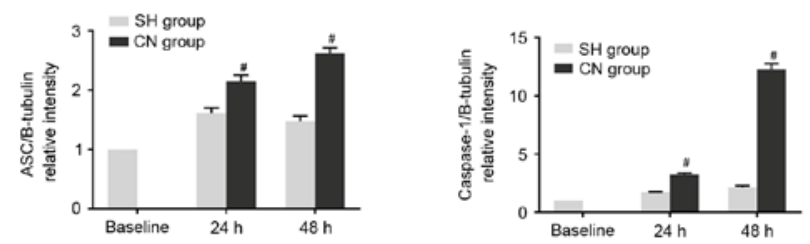

H

Figure 4. (A) Rectal temperature was lower in the $\mathrm{SH}$ group than in the $\mathrm{CN}$ group. ${ }^{*} \mathrm{P}<0.05$ vs. CN Group I; ${ }^{\text {P }}<0.05$ vs. CN Group II. (B) Neurologic deficit scale was higher in the $\mathrm{SH}$ group than in the $\mathrm{CN}$ group. ${ }^{\text { }} \mathrm{P}<0.05$ vs. $\mathrm{CN}$ group. (C) Serum $\mathrm{S} 100 \mathrm{~B}$ concentration was lower in the $\mathrm{SH}$ group than in the $\mathrm{CN}$ group. ${ }^{\#} \mathrm{P}<0.05$ vs. CN group. (D) Expression levels of NLRP3, ASC, caspase-1 and caspase-3 were observed to significantly decrease in the SH compared with the CN groups. Statistical analysis of the relative intensity of (E) NLRP3, (F) ASC, (G) caspase-1 and (H) caspase-3 expression. "P<0.05 vs. Baseline control group (unpaired Student's t-test). SH, spontaneous hypothermia; CN, controlled normothermia; S100B, S100 calcium-binding protein B; NLRP3, NACHT, LRR, and PYD domains-containing protein 3; ASC, apoptosis-associated speck-like protein containing a CARD. 

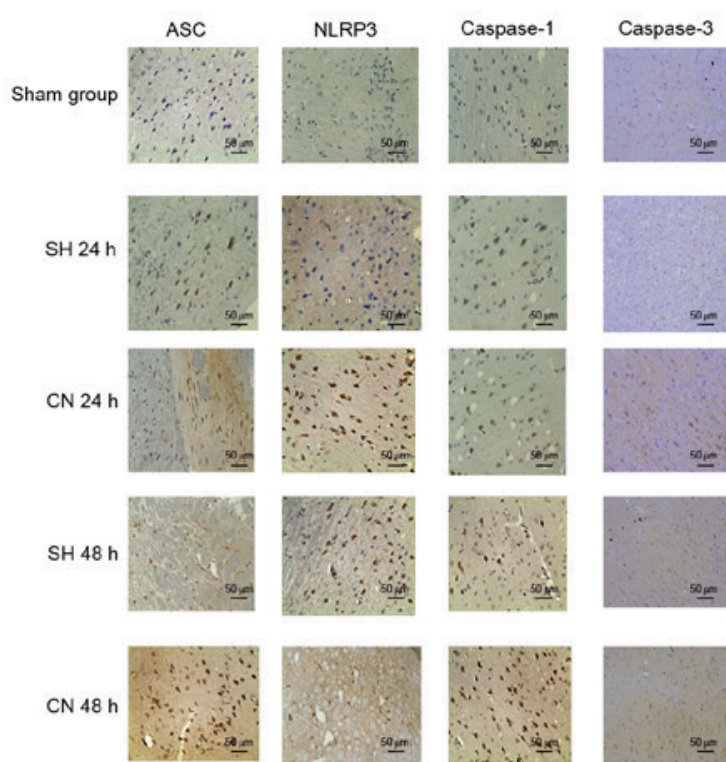
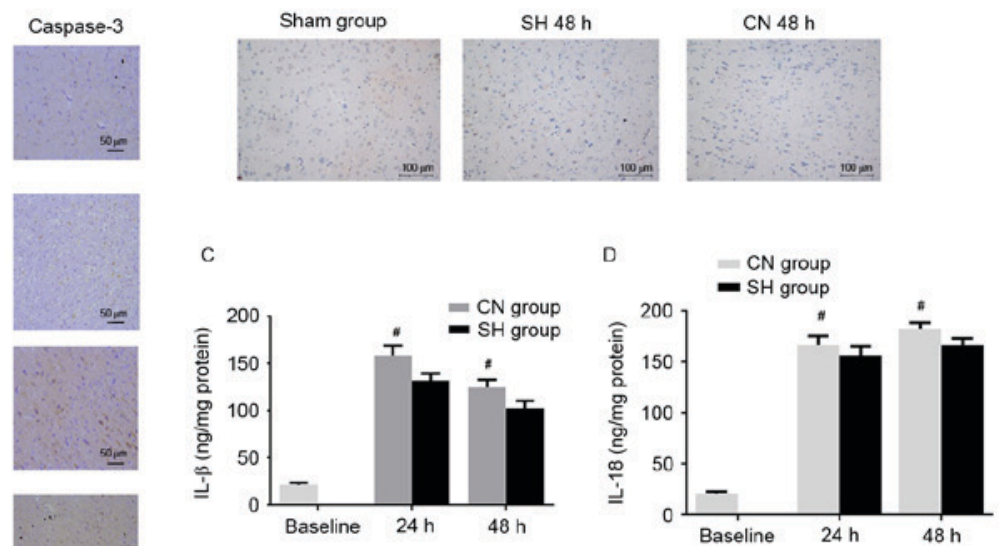

E
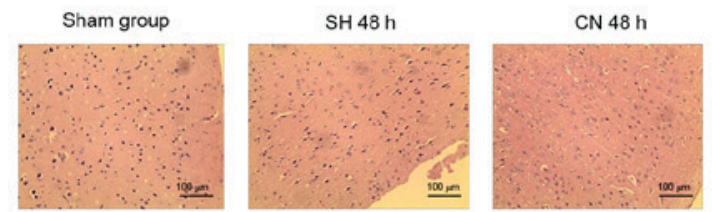

Figure 5. (A) Immunohistochemistry indicated that the number of ASC, NLRP3, caspase-1 and caspase-3 positive cells in the cerebral cortex increased in the CN group compared with the SH group. Scale bar, $50 \mu \mathrm{m}$. (B) A small number of TUNEL-positive cells was observed in the cerebral cortex of the control (Sham) group. The number of TUNEL positive cells was lower in the SH group compared with the CN group. Scale bar, $100 \mu \mathrm{m}$. The (C) IL-1 $\beta$ and (D) IL-18 concentrations were decreased in the SH group compared with the $\mathrm{CN}$ group. (E) Mild damage was observed in the control (Sham) group, while the damage was more severe in the $\mathrm{CN}$ group compared with the SH group. Scale bar, $100 \mu \mathrm{m} .{ }^{~} \mathrm{P}<0.05$ vs. CN group (unpaired Student's t-test). ASC, apoptosis-associated speck-like protein containing a CARD; NLRP3, NACHT, LRR, and PYD domains-containing protein 3; CN, controlled normothermia; SH, spontaneous hypothermia; IL, interleukin.

that expression levels of caspase- 1 and caspase- 3 decreased with $\mathrm{SH}$ in CA models. This result was similar to that observed in the hippocampus, where caspase- 1 and caspase- 3 expression increased following asphyxia. In recent years, combination therapy has been used to ameliorate neural disorders (31). Therefore, the present study provides solid evidence to support the potential clinical application of hypothermia. Future investigations will focus on survival prognosis, in order to determine whether hypothermia completely replaces the standard treatment for CA-induced neurologic deficits.

In conclusion, the present study demonstrates that $\mathrm{SH}$ ameliorated inflammation and neurologic deficit in CA models following resuscitation. The findings are important to increase understanding of the underlying mechanisms in CA-induced inflammation and neurologic damage following ROSC. Furthermore, the current findings promote a potential novel therapeutic strategy, which may be a promising candidate for increasing the survival rate and quality of life for patients suffering from post-CA syndrome.

\section{Acknowledgements}

The current study was supported by a research grant from project of Leading Talents in the Pearl River Talent Plan of Guangdong Province (grant no. 81000-42020004).

\section{References}

1. Berdowski J, Berg RA, Tijssen JG and Koster RW: Global incidences of out-of-hospital cardiac arrest and survival rates: Systematic review of 67 prospective studies. Resuscitation 81 : $1479-1487,2010$
2. Hua W, Zhang LF, Wu YF, Liu XQ, Guo DS, Zhou HL, Gou ZP, Zhao LC, Niu HX, Chen KP, et al: Incidence of sudden cardiac death in China: Analysis of 4 regional populations. J Am Coll Cardiol 54: 1110-1118, 2009.

3. Nichol G, Stiell IG, Laupacis A, Pham B, Maio VJ and Wells GA: A cumulative meta-analysis of the effectiveness of defibrillator-capable emergency medical services for victims of out-of-hospital cardiac arrest. Ann Emerg Med 34: 517-525, 1999.

4. Wang HE, Min A, Hostler D, Chang CC and Callaway CW: Differential effects of out-of-hospital interventions on short- and long-term survival after cardiopulmonary arrest. Resuscitation 67: 69-74, 2005.

5. Laver S, Farrow C, Turner D and Nolan J: Mode of death after admission to an intensive care unit following cardiac arrest. Intensive Care Med 30: 2126-2128, 2004.

6. Nolan JP, Laver SR, Welch CA, Harrison DA, Gupta V and Rowan K: Outcome following admission to UK intensive care units after cardiac arrest: A secondary analysis of the ICNARC Case Mix Programme Database. Anaesthesia 62: 1207-1216, 2007.

7. Neumar RW: Molecular mechanisms of ischemic neuronal injury. Ann Emerg Med 36: 483-506, 2000.

8. Johnson EM Jr, Greenlund LJ, Akins PT and Hsu CY: Neuronal apoptosis: Current understanding of molecular mechanisms and potential role in ischemic brain injury. J Neurotrauma 12: 843-852, 1995.

9. Mizushima H, Zhou CJ, Dohi K, Horai R, Asano M, Iwakura Y, Hirabayashi T, Arata S, Nakajo S, Takaki A, et al: Reduced postischemic apoptosis in the hippocampus of mice deficient in interleukin-1. J Comp Neurol 448: 203-216, 2002.

10. Lu A and $\mathrm{Wu} \mathrm{H}$ : Structural mechanisms of inflammasome assembly. FEBS J 282: 435-444, 2015.

11. Martinon F: Detection of immune danger signals by NALP3 J Leukoc Biol 83: 507-511, 2008.

12. Rathinam VA, Vanaja SK and Fitzgerald KA: Regulation of inflammasome signaling. Nat Immunol 13: 333-342, 2012.

13. Shigeoka AA, Mueller JL, Kambo A, Mathison JC, King AJ, Hall WF, Correia Jda S, Ulevitch RJ, Hoffman HM and McKay DB: An inflammasome-independent role for epithelial-expressed Nlrp3 in renal ischemia-reperfusion injury. J Immunol 185: 6277-6285, 2010. 
14. Xu Y, Sheng H, Bao Q, Wang Y, Lu J and Ni X: NLRP3 inflammasome activation mediates estrogen deficiency-induced depression- and anxiety-like behavior and hippocampal inflammation in mice. Brain Behav Immun 56: 175-186, 2016.

15. Zendedel A, Johann S, Mehrabi S, Joghataei MT, Hassanzadeh G, Kipp $\mathrm{M}$ and Beyer C: Activation and regulation of NLRP3 Inflammasome by Intrathecal application of SDF-1a in a spinal cord injury model. Mol Neurobiol 53: 3063-3075, 2016.

16. Liu HD, Li W, Chen ZR, Hu YC, Zhang DD, Shen W, Zhou ML, Zhu L and Hang CH: Expression of the NLRP3 inflammasome in cerebral cortex after traumatic brain injury in a rat model. Neurochem Res 38: 2072-2083, 2013.

17. Yang F, Wang Z, Wei X, Han H, Meng X, Zhang Y, Shi W, Li F, Xin T, Pang Q and Yi F: NLRP3 deficiency ameliorates neurovascular damage in experimental ischemic stroke. J Cereb Blood Flow Metab 34: 660-667, 2014.

18. Liu L and Yenari MA: Therapeutic hypothermia: Neuroprotective mechanisms. Front Biosci 12: 816-825, 2007.

19. Gong P, Hua R, Zhang Y, Zhao H, Tang Z, Mei X, Zhang M, Cui J and Li C: Hypothermia-induced neuroprotection is associated with reduced mitochondrial membrane permeability in a swine model of cardiac arrest. J Cerebr Blood Flow Metad 33: 928-934, 2013

20. Rubiano AM, Sanchez AI, Estebanez G, Peitzman A, Sperry J and Puyana JC: The effect of admission spontaneous hypothermia on patients with severe traumatic brain injury. Injury 44: $1219-1225,2013$

21. Hickey RW, Ferimer H, Alexander HL, Garman RH, Callaway CW, Hicks S, Safar P, Graham SH and Kochanek PM: Delayed, spontaneous hypothermia reduces neuronal damage after asphyxial cardiac arrest in rats. Crit Care Med 28: 3511-3516, 2000.

22. D'Cruz BJ, Logue ES, Falke E, DeFranco DB and Callaway CW: Hypothermia and ERK activation after cardiac arrest. Brain Res 1064: 108-118, 2005.

23. Jia X, Koenig MA, Shin HC, Zhen G, Pardo CA, Hanley DF, Thakor NV and Geocadin RG: Improving neurological outcomes post-cardiac arrest in a rat model: Immediate hypothermia and quantitative EEG monitoring. Resuscitation 76: 431-442, 2008.
24. Drabek T, Janata A, Wilson CD, Stezoski J, Janesko-Feldman K, Tisherman SA, Foley LM, Verrier JD and Kochanek PM: Minocycline attenuates brain tissue levels of TNF- $\alpha$ produced by neurons after prolonged hypothermic cardiac arrest in rats. Resuscitation 85: 284-291, 2014.

25. Kida K, Shirozu K, Yu B, Mandeville JB, Bloch KD and Ichinose F: Beneficial effects of nitric oxide on outcomes after cardiac arrest and cardiopulmonary resuscitation in hypothermia-treated mice. Anesthesiology 120: 880-889, 2014.

26. Vijlbrief DC, Benders MJ, Kemperman H, van Bel F and de Vries WB: Cardiac biomarkers as indicators of hemodynamic adaptation during postasphyxial hypothermia treatment. Neonatology 102: 243-248, 2012.

27. Callaway CW, Rittenberger JC, Logue ES and McMichael MJ: Hypothermia after cardiac arrest does not alter serum inflammatory markers. Crit Care Med 36: 2607-2612, 2008.

28. Ding L, Gao X, Yu S and Yang J: Effects of mild and moderate hypothemia therapy on expression of cerebral neuron apoptosis related proteins and glial fiber acidic protein after rat cardio-pulmonary resuscitation. Cell Biochem Biophys 70: 1519-1525, 2014.

29. Lu J, Shen Y, Qian HY, Liu LJ, Zhou BC, Xiao Y, Mao JN, An GY, Rui MZ, Wang T and Zhu CL: Effects of mild hypothermia on the ROS and expression of caspase-3 mRNA and LC3 of hippocampus nerve cells in rats after cardiopulmonary resuscitation. World J Emerg Med 5: 298-305, 2014.

30. Thornberry NA and Lazebnik Y: Caspases: Enemies within. Science 281: 1312-1316, 1998.

31. Wiklund L, Zoerner F, Semenas E, Miclescu A, Basu S and Sharma HS: Improved neuroprotective effect of methylene blue with hypothermia after porcine cardiac arrest. Acta Anaesth Scand 57: 1073-1082, 2013.

This work is licensed under a Creative Commons Attribution-NonCommercial-NoDerivatives 4.0 International (CC BY-NC-ND 4.0) License. 